\title{
The Diminution of Variation in Bacterial Populations with Special Reference to Klebsiella pneumoniae and Drug Resistance
}

\author{
BY W. HOWARD HUGHES \\ Wright-Fleming Institute, St Mary's Hospital Medical School, \\ Paddington, London, W. 2
}

(Received 25 November 1960)

\section{SUMMARY}

Single organisms of Klebsiella pneumoniae were repeatedly selected for resistance on streptomycin agar yielding a population with increased resistance clearly different from that of the parent strain. These two strains were grown together and when retested a uniform population was found to be present with streptomycin resistance intermediate between the initial two.

Two strains of Klebsiella pneumoniae, one the type-strain, the other a chloramphenicol-resistant mutant with a different colonial appearance, were grown together and the mixture sampled at intervals. The two populations became progressively more alike until within six hours they were indistinguishable. The resulting uniform population was intermediate in sensitivity to chloramphenicol and the colonies could no longer be assigned easily to either parental type on morphological grounds. When the two strains were separated by a collodion membrane, this diminution of variation did not occur.

The results cannot be explained by overgrowth of one strain by the other. It is suggested that something analogous to inbreeding is taking place.

\section{INTRODUCTION}

Previous work has shown that individual bacteria within clones differ from one another (Hughes, 1952, 1953, 1955 $a, b$ ). This type of variation is continuous and more common than mutation as ordinarily understood. This work was summarized by Hughes (1957), and the conclusions reached agree in the main with the view advanced by Yudkin (1953) based on his own studies about acquired drug resistance. The subject has received mathematical consideration from Powell (1956, 1958).

The following work was undertaken to find out why, if variation could be found between the individuals, the population as a whole remained stable. In an organism in which sexual reproduction is recognized this is no problem, since any major variation or mutation which is not lethal will be distributed by random crossing. The predominant views on bacterial reproduction predispose against accepting a similar mechanism among cells which multiply by so-called simple fission.

It has now been found that when two populations, derived from the same source but which are different in regard to resistance to streptomycin, chloramphenicol and other characters, are grown together for a short time, a uniform population inter- 
mediate in respect to the characters studied emerges very rapidly. This diminution of variation appears to differ in its general properties from the changes associated with established parasexual mechanisms, such as transduction, transformation or conjugation.

\section{METHODS}

Organism. A strain of Klebsiella pneumoniae (NCTC 7242) first obtained in 1946 for the assay of streptomycin was used. This had been repeatedly subcultured.

Isolation of single organisms. A De Fonbrune micromanipulator and a cheap modification of his oil chamber (made by Hawksley and Sons Ltd., London) were used throughout this series of experiments.

Experimental procedures. Young cultures were obtained by inoculating one loopful of the stock culture stored on the bench into nutrient broth in a screw-capped bottle. This was clipped to a drum rotating at $8 \mathrm{rev} . / \mathrm{min}$. in an incubator at $37^{\circ}$ and left for $3 \mathrm{hr}$. One $3.5 \mathrm{~mm}$. loopful of this subculture was spread on a block of agar about $1.0 \mathrm{~cm}$. square mounted on the cover glass of the oil chamber. After air drying the coverslip was reversed and the oil introduced. The specimen was then incubated, usually for $3 \mathrm{hr}$., and the size of individual colonies recorded. In the presence of streptomycin or chloramphenicol, the colony diameter is an index of the average resistance of the organisms of the colony to the concentration of antibiotic in the agar. Measurements in arbitrary units were made with an eye-piece micrometer (total magnification usually 240 diameters); 100 colonies were measured and the histogram of the population plotted. Any colonies which had fused were discarded from the count.

The planting out of single organisms was impracticable since the time lag between the first and the last isolations would invalidate the investigation. On special occasions the specimens were examined when only one or two divisions had taken place and any colonies found to be too near to one another were decreased in number by removing those likely to come into contact. This was not usually necessary since, with the small implants used less than $5 \%$ of the colonies would have been involved and these could have been recognized and excluded after incubation.

From the most rapidly growing colonies single organisms were picked and grown on to give strains which were again tested for their rates of growth at the same concentration of antibiotic. Those found to be growing better than the previous strains were retained as having a higher resistance. This sequence was repeated until a strain had been selected which grew as well on antibiotic agar as the parent strain on plain nutrient agar. It had already been demonstrated that single streptomycinresistant cells of Klebsiella pneumoniae could be selected and stable strains obtained (Hughes, 1957) as had been done previously with staphylococci with regard to penicillin (Hughes, 1952; Eagle, Fleischman \& Levy, 1952).

Having obtained two strains which could be separated by the rates of growth of their colonies in the presence of antibiotic it became possible to reconsider Yudkin's hypothesis on populations of bacteria (Yudkin, 1953). Clearly if differences between organisms could be demonstrated in small populations, then in large populations a great diversity should occur; experience does not support this and there must be some mechanism to counter excessive variation. 


\section{RESULTS}

Experiment 1 was designed to demonstrate whether or not the two strains grown together would influence one another's resistance to antibiotic. A loopful of a $3 \mathrm{hr}$. broth culture of the original strain and a similar inoculum of the 17th single cell subculture (each of which had been selected for increased resistance) were separately planted on $1.0 \mathrm{~cm}$. square blocks cut from a poured nutrient agar plate containing $10 \mu \mathrm{g} . / \mathrm{ml}$. of streptomycin. An equal volume of a $17 \mathrm{hr}$. mixed culture of the two strains diluted to the equivalent concentration was similarly seeded. The three blocks were arranged in the same oil chamber, covered with sterile paraffin oil and incubated at $37^{\circ}$ for $3 \mathrm{hr}$., when the colony diameters were measured.

The resulting histograms are shown in Fig. 1; after incubation together in mixed culture instead of the two peaks characterizing the separate cultures, only a uniform population was present, the average colony diameter falling between the two extremes but, on an arithmetic scale, nearer to the size of the 'sensitive' colony. This strongly suggested that the two populations had, if not 'interbred', at least influenced one another, and further study of the phenomenon was made.

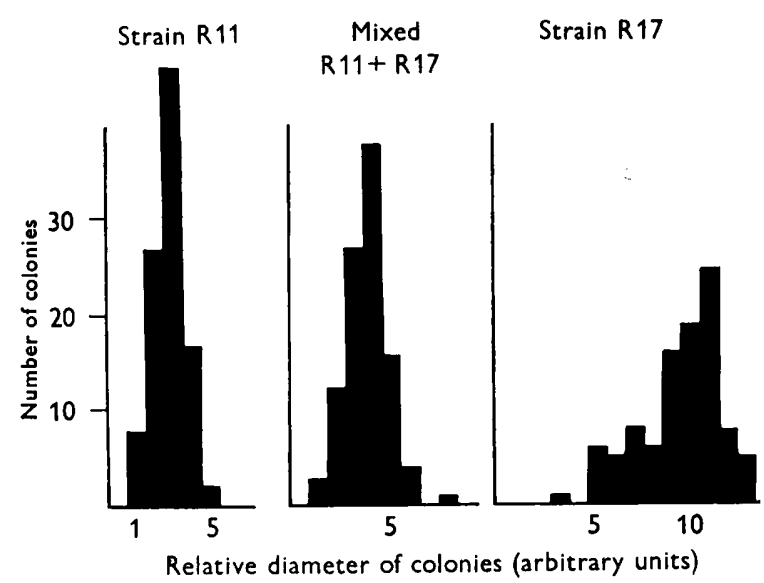

Fig. 1. Histograms showing distribution of size among colonies, growing for $3 \mathrm{hr}$. on agar containing streptomycin $(10 \mu \mathrm{g} . / \mathrm{ml}$.), derived from a streptomycin-sensitive strain of Klebsiella pneumoniae NCTC 7242 (R11) and a more resistant strain derived from it (R 17). Colony diameter measured in arbitrary units.

Experiment 2. Certain difficulties had been apparent in the earlier experimental arrangements. For example, with streptomycin it was only possible to select for resistance, not for sensitivity, since streptomycin is bactericidal and the most susceptible members of all the populations were lost. Further, streptomycin was unstable under the conditions in which the agar blocks were prepared. In all subsequent experiments freshly prepared chloramphenicol solutions were used.

Into a dense suspension of Klebsiella pneumoniae an equal volume of broth containing chloramphenicol was poured so that the final concentration of chloramphenicol was near the inhibitory concentration for small implants, in this case $8 \mathrm{units} / \mathrm{ml}$. The mixture was incubated overnight and then planted as a wash on a ditch plate containing 30 units chloramphenicol in the ditch of a Petri dish con- 
taining $15 \mathrm{ml}$. agar medium. One single colony was found near the ditch. The strain so obtained (KR) was compared with one of the sensitive strains (K5) chosen for its neat colony form and reliable cultural characteristics. A mixed culture of the two strains in broth at $37^{\circ}$, was sampled at intervals, diluted in broth and planted on agar blocks containing chloramphenicol ( 8 units $/ \mathrm{ml}$.). The filled oil chambers were cooled at $4^{\circ}$ until the full series had been collected. They were incubated overnight

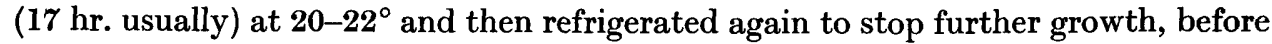
being measured.
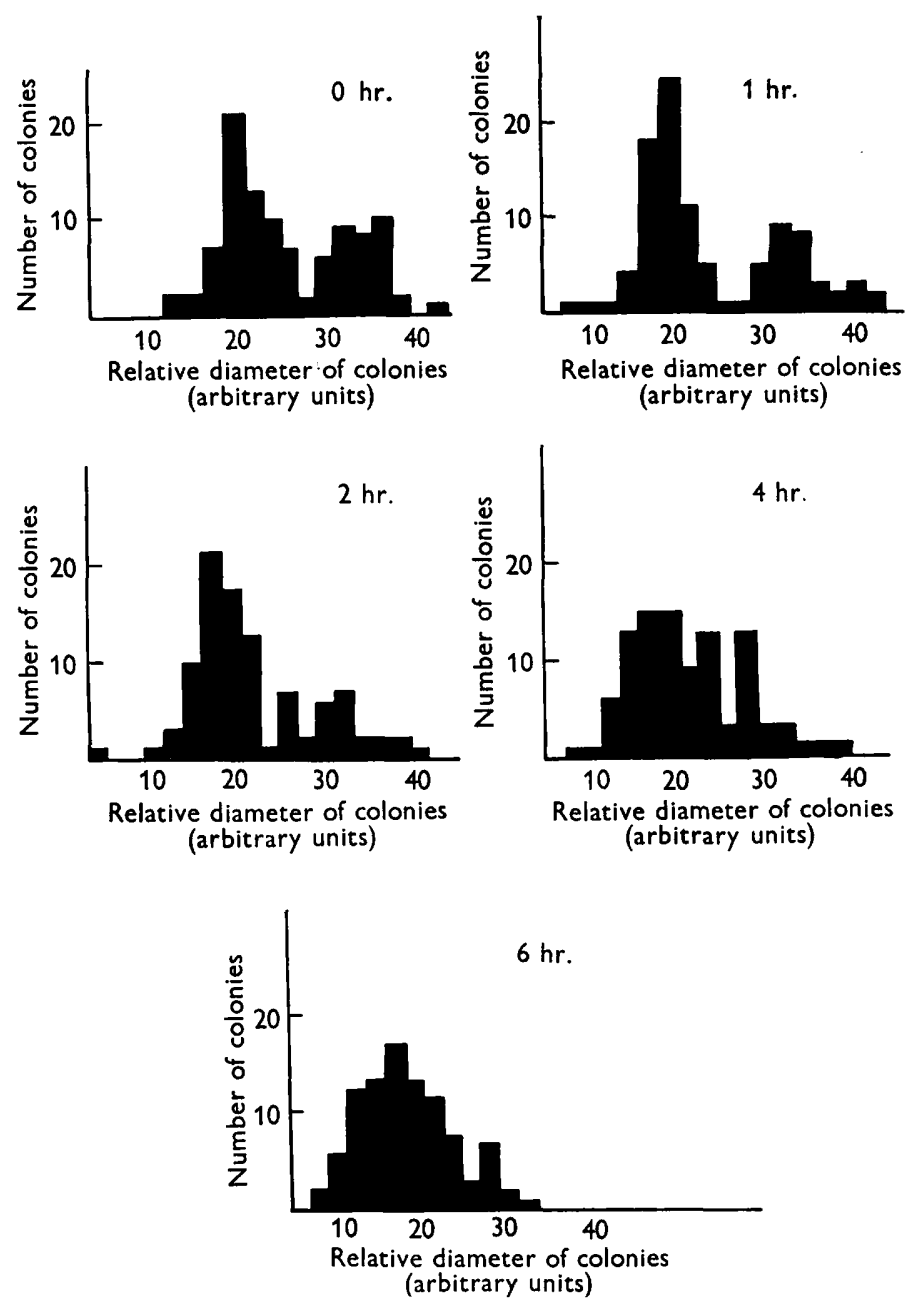

Fig. 2. Histograms showing distribution of size among colonies, growing overnight at $20-22^{\circ}$ on agar containing chloramphenicol (8 units $\left./ \mathrm{ml}\right)$, derived from a mixed culture of a sensitive (K5) and a resistant (KR) strain of Klebsiella pneumoniae. Samples were plated at $0,1,2,4$ and $6 \mathrm{hr}$. after mixing.

The histograms in Fig. 2 make clear the transition from two independent populations to one which was uniform. The whole cycle with a stationary culture appeared to be complete in only $6 \mathrm{hr}$. It was fortunate that the resistant strain here used was 
distinguishable from the sensitive strain. The resistant strain produced much more slime and capsular material than did the sensitive strain; broth cultures were sticky and tenacious when touched with a wire loop. The individual colonies of the resistant strain at all stages of growth were recognizably different from the sensitive colonies. The resistant colonies were irregular in outline with a coarser surface texture, whereas the sensitive were circular and uniformly smooth.

During incubation with the sensitive strain the size of the colonies of the resistant strain in the samples became progressively smaller and many were morphologically intermediate in type so that while at first it was easily possible to score the two strains separately, later the characters were so mixed as to make this difficult. In Fig. 2, as in other diagrams, not all of the results available are shown; the readings at 3, 5, 7 and $8 \mathrm{hr}$., however, are in complete accord. A number of controls had to be set up before it could be stated that the change of size, that is the alteration of resistance, was due to the influence of the one strain on the other.

Experiment 3. In order to discover whether the change in average size of colony was due to an overgrowing of one strain by the other in broth culture, the opacity of growing broth cultures of the two strains, separately and together, was measured with a Hilger-Watts Spekker absorptiometer.

The results are shown in Fig. 3; the resistant strain evidently had a longer lag period. When an old culture was used there would therefore be a delay in growth as compared with the sensitive strain, and this would diminish the differences between the two. This difficulty was overcome when cultures already grown in broth for $3 \mathrm{hr}$. were used.

Experiment 4. To find whether the conditions for growth in the oil chamber favoured one strain more than the other the two were grown both separately and together on plain agar. Under these conditions the sensitive strain formed the larger colonies but both grew well. When equal volumes of young cultures of the two strains were planted together in plain broth and sampled at intervals on to agar, it was found that the ratio of the one to the other did not alter appreciably during incubation. The strains could be differentiated from one another by the colony morphology, at least during the first $6 \mathrm{hr}$. of incubation. This observation agrees well with the results of the opacity readings in Fig. 3.

Experiment 5. To show whether actual contact is needed between the cells of the two strains in order to bring about the changes seen when they are grown together, the standard experiment was carried out with the two strains separated from each other by a collodion (Gradocol) membrane of average pore size $0.46 \mu$. The resulting histograms do not suggest that there was any significant change (Fig. 4).

Experiment 6. It was decided to investigate the effect of disturbance on the cultures. The mixture of organisms was put up as before but in tubes which were rocked in a water bath at $37^{\circ}$ with about 45 oscillations/min. Samples were taken as before. The preparations were inspected to exclude any possible aggregation into pairs, so that the colonies measured should not be the product of conjoined pairs of organisms. The results are shown in Fig. 5. Again it will be seen that the same trend was present, the shaking merely accelerated the result. A technical difficulty that has not yet been overcome may be mentioned here. Materials for an experiment are prepared and the organisms planted and incubated in broth. Samples withdrawn at predetermined intervals are transferred to the agar medium in oil chambers and 
stored in the refrigerator. The results obtained on subsequent culture depend, if they are to be compared, on the starting temperature of each oil chamber being identical and on all the organisms remaining equally in the log phase of growth. When sampling is at hourly intervals, then the rate of equilibration is important; the $7 \mathbf{~ h r}$. specimen will be exposed for only $30 \mathrm{~min}$. at $4^{\circ}$ while the earlier ones will have had I.5, $2.5 \mathrm{hr}$., respectively, and so on. It appears from our results that something of

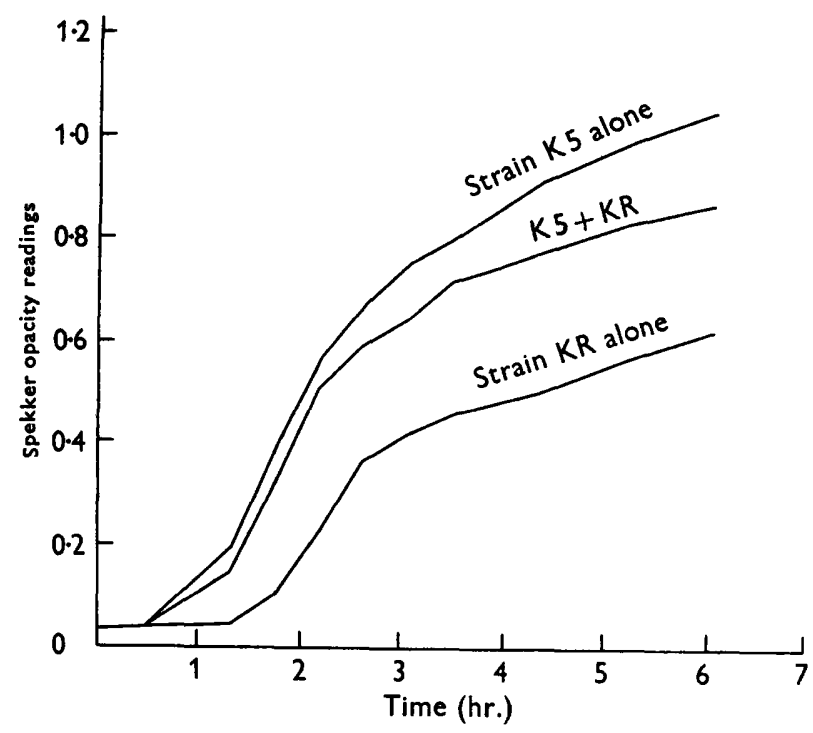

Fig. 3. Growth curves of sensitive strain (K5) and resistant mutant (KR) of Klebsiella pneumoniae, in separate and mixed broth culture at $37^{\circ}$. Opacity readings were made with a Spekker absorptiometer.
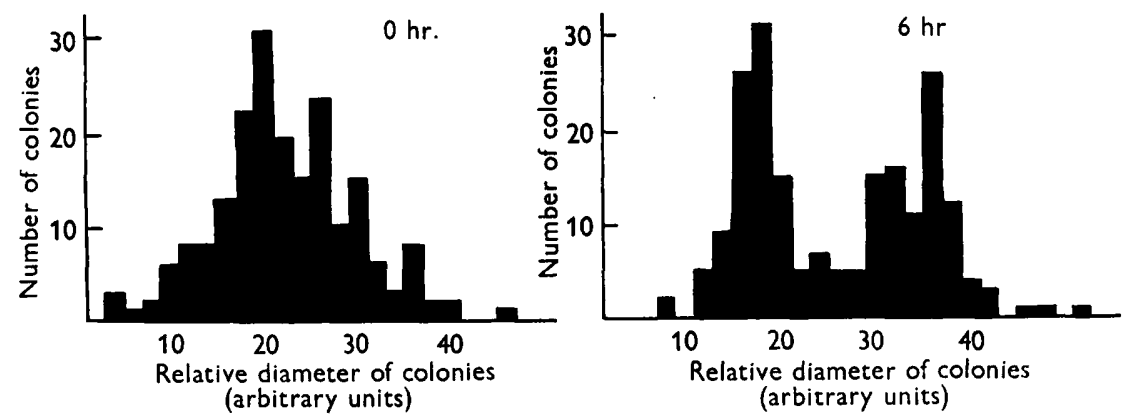

Fig. 4. Histograms showing the distribution of the size of colonies of the sensitive (K5) and resistant (KR) strains of Klebsiella pneumoniae, grown in broth and separated from each other by a Gradocol membrane.

the order of cooling for $3 \mathrm{hr}$. is desirable in the English summer. This conclusion was based on the following observation. A micromanipulator cell, filled with oil containing the agar block was connected to a thermocouple inside a refrigerator, the galvanometer being on a support outside. It was found that while cooling from room temperature began rapidly and evenly, at the lower range temperature fluctuations were marked and a settled low value was not reached until at least $3 \mathrm{hr}$. By 
this time the organisms of the early samples would have passed into the lag phase and all late samples in the series will appear to grow more rapidly than the earlier. This might be overcome by refrigerating for a prolonged period when all samples would show the same lag.
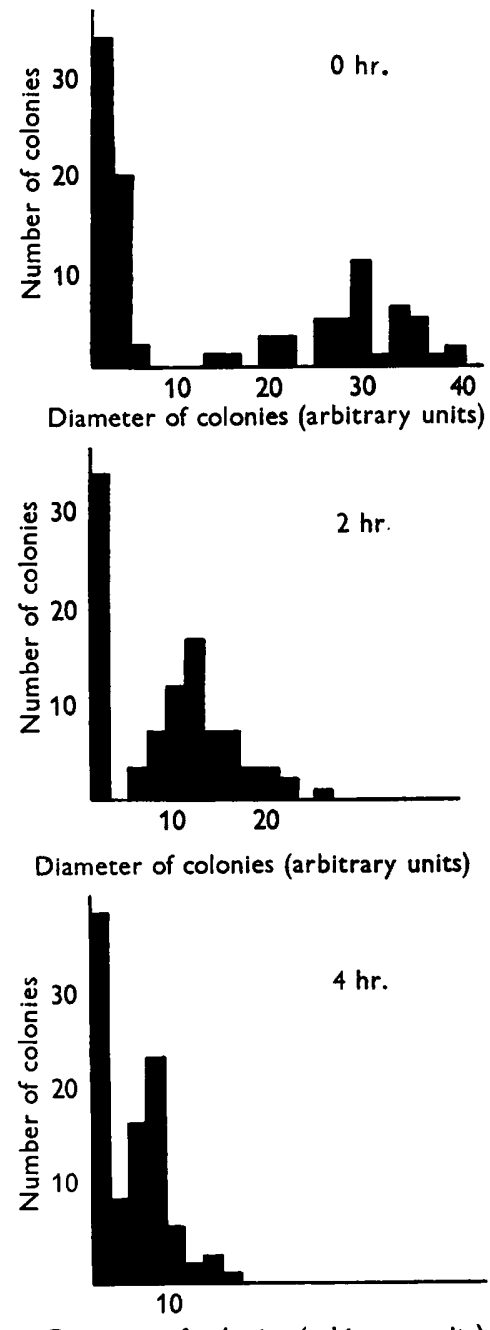

Fig. 5. Histograms showing the distribution of colony size as before. Sensitive and resistant strains of Klebsiella pneumoniae grown together in a broth culture, rocked at about 45 oscillations/min. in a water bath at $37^{\circ}$, and sampled on the 8 units chloramphenicol/ml. agar.

\section{DISCUSSION}

It has long been recognized that many drug-resistant 'mutants' selected by whatever method tend to alter on subculture, becoming rather more sensitive than when first isolated. This can be explained easily enough if it be supposed that the colony taken contains a mixture of organisms which, when in subculture, influence one 
another towards a mean. In the present experiments two strains both derived from the same pure culture of Klebsiella pneumoniae and grown repeatedly from single organisms, when incubated together merged progressively into a uniform population. This disappearance of the mutant strain or, in the first series of experiments where streptomycin was used, of the clone selected for streptomycin-resistance, was not due to overgrowth by the sensitive strain, for, when the growth rates were balanced by using only actively growing cultures, the ratio of the numbers of one strain to the other remained constant throughout the experiment.

That the strains did not influence one another when separated by a coarse grade of collodion membrane through which any large molecule would pass easily suggests that actual contact between the organisms may be necessary. Relatively gentle agitation only accelerated the process and did not at all inhibit it. A suitable technique for demonstrating the results of violent agitation such as is needed to separate recombining Escherichia coli organisms (Wollman, Jacob \& Hayes, 1956) has not been devised for the present work.

A demonstration of the kind given here seems necessary as a corollary to Yudkin's work and in particular to his theoretical considerations about bacterial populations and the emergence of resistant strains. Yudkin pointed out (1953; Fig. 2) that if cells divided 'unequally' then a population would be obtained which would be progressively variable when grown in the absence of a drug (or of other selecting mechanism) until, after many subcultures, the permanent form of the distribution curve would be reached. For one character, e.g. drug resistance, there is obviously a limit to variation; finality is reached when the population contains some completely resistant individuals. For all the factors that make up the complex genetic pattern of the population, almost infinite variation is possible. Nevertheless, since various microbial diseases described by ancient writers are still recognizable today, their causal organisms cannot have greatly changed. Similarly, when the Oxford staphylococcus was collected from a number of centres and re-examined after about 10 years' use as a standard, variation was within the limits of the assay methods in use. This is no longer true, however; it is probable that substitution of other strains has now taken place (Oeding \& Ostervold, 1959).

It seems probable that the relative uniformity of members of microbial populations depends on regular interchange of hereditary material. Under the highly artificial conditions of laboratory culture on solid media, or where deliberate selection with chemicals or otherwise takes place, pressures will be brought to bear on the population which will disclose its latent heterogeneity. Usually variations can be detected only in early divisions of the organisms on solid medium; once they have been separated as isolated colonies, no further variation takes place. This was noticed in the earlier experiments with spontaneously occurring long forms of Escherichia coli (Hughes, 1953). Progressive homogenization of a mixture of strains has been found repeatedly, irrespective of the time of incubation or the concentration of antibiotic used for the detection of strain differences.

\section{REFERENCES}

Eagle, H., Fleischman, R. \& Levy, M. (1952). Development of increased bacterial resistance to penicillin, chloramphenicol and streptomycin. 1. Continuous spectrum of resistance to penicillin, chloramphenicol and streptomycin. J. Bact. 63, 623. 
Hughes, W. H. (1952). Variation in penicillin resistance in single-cell cultures of Staphylococcus aureus. J. gen. Microbiol. 6, 175.

Hughes, W. H. (1953). The origin of the L-form variants in anaerobic cultures of Bacterium coli. J. gen. Microbiol. 8, 307.

Hughes, W. H. (1955a). The inheritance of differences in growth rate in Escherichia coli. J. gen. Microbiol. 12, 265.

Hughes, W. H. (1955b). The differences in antibiotic sensitivity of closely related single cells of Proteus vulgaris. J. gen. Microbiol. 12, 269.

Hughes, W. H. (1957). Inheritance in single bacterial cells. Ciba Foundation Symposium on Drug Resistance in Micro-organisms, pp. 64-71.

Oeding, P. \& Ostervold, B. (1959). The stability of Oxford Staph. H. and other penicillin reference strains. Acta path. microbiol. scand. 46, 149.

Powell, E. O. (1956). Growth rate and generation time of bacteria, with special reference to continuous culture. J. gen. Microbiol. 15, 492.

PoweLl, E. O. (1958). An outline of the pattern of bacterial generation times. J. gen. Microbiol. 18, 382.

Wollman, E. L., JACOB, F. \& HAYES, W. (1956). Conjugation and genetic recombination in Escherichia coli K-12. Cold Spr. Harb. Symp. quant. Biol. 21, 141.

YUDKIN, J. (1953). Origin of acquired drug resistance in bacteria. Nature, Lond. 171, 541. 